\title{
Liquid Cell TEM Studies of Galvanic Displacement Reactions in Aqueous Solutions
}

\author{
Jeung Hun Park ${ }^{1,2}$, Daniel A. Steingart ${ }^{3}$, Lili Deligianni ${ }^{1}$, Suneel Kodambaka ${ }^{2}$, and Frances M. Ross ${ }^{1}$ \\ 1. IBM T. J. Watson Research Center, Yorktown Heights, NY 10598, United States. \\ 2. Department of Materials Science and Engineering, University of California Los Angeles, \\ Los Angeles, CA 90095, United States. \\ 3. Department of Mechanical and Aerospace Engineering, and Andlinger Center for Energy \\ and the Environment, Princeton University, Princeton, NJ 08544, United States.
}

Galvanic displacement reactions are a versatile approach to achieving sophisticated nanostructure engineering. One key application is the formation of nanopatterned surfaces, such as Ni decorated with Pd nanoparticles, which act as highly catalytic substrates for subsequent electroless deposition [1]. Another application is the formation of magnetic nanoparticles with axial or radial compositional modulation [2] and a variety of applications [3]. Precise engineering of the final morphology relies on a deep understanding of the reaction pathways involved. Although ex situ microscopies provide critical information, the ability to record the process as it takes place is expected to contribute to a deeper understanding [4]. Liquid cell TEM, especially when coupled with electrochemical control and analytical capabilities, provides the needed tool for measuring structural and chemical changes in liquids [4-6]. Here, we use in situ liquid cell TEM to examine a model system, galvanic deposition of Pd nanoparticles on $\mathrm{Ni}$ in an acidic solution, and extend our materials system toward other metal films.

We examined galvanic displacement reactions in samples consisting of 20-50 nm thick Ni films deposited by electron beam evaporation onto a $50 \mathrm{~nm}$ thick silicon nitride membrane that makes up one viewing window of the liquid cell. These films were imaged in a FEI CM30 TEM, operated at $300 \mathrm{kV}$ [7], in a continuous flow Hummingbird Scientific LC-TEM holder [8, 9]. Deionized water was introduced into the liquid cell to remove air bubbles, after which a solution was introduced containing $0.1 \mathrm{M} \mathrm{HCl} \mathrm{or} \mathrm{H}_{2} \mathrm{SO}_{4}$ with ppm levels of $\mathrm{PdSO}_{4}$. We used low dose imaging protocols and verified that the solutions can be examined for long periods (hours) without visible beam effects. We observe two different reactions. At long times, we see corrosion of the Ni film under the beam, presumably due to reactive species produced by radiolysis. Figure 1 presents slow Ni corrosion in deionized water $(\mathrm{pH}=7)$ under irradiation, in an example where $\mathrm{PdSO}_{4}$ is not introduced. This process took place at a flow rate of $5 \mu \mathrm{l} / \mathrm{min}$ and zero voltage applied to the electrode. The electron beam causes dissolution by creating reactive radiolytic species in water, i.e. hydrated electrons and radicals, which convert Ni to ions [5, 6, 9]. Dissolution appears to take place via the general corrosion mode (Fig. 1a). To obtain quantitative reaction kinetics, the average image brightness was analyzed as a function of irradiation time (Fig. 1b), showing an induction time (Fig. 1c). The second reaction observed is the galvanic displacement reaction itself (Figure 2). Here, nucleation and growth of $\mathrm{Pd}$ particles tens of $\mathrm{nm}$ in diameter is visible when $0.1 \mathrm{M} \mathrm{H}_{2} \mathrm{SO}_{4}(\mathrm{pH}=1)$ with ppm levels of $\mathrm{PdSO}_{4}$ is flowed (Fig. 2c). Simultaneously, Ni dissolution takes place. Compared to the reaction without the Pd additive, Ni dissolution is accelerated in the presence of Pd; this is as expected since Ni goes into solution as Pd precipitates (Fig. 2a). In the example shown, preferential dissolution is visible at grain boundaries. The dissolution rate increases with the flow rate (Fig. 2b). Solution $\mathrm{pH}$ and additives also change the overall reaction rate of the film.

We will describe the kinetics of these processes in solutions of different $\mathrm{pH}$ and $\mathrm{Pd}$ concentration, and also discuss corrosion reactions in other metals, $\mathrm{Cu}, \mathrm{Au}$, and Pt. Measurements of the local variations morphology, composition, nucleation sites (grain boundaries vs. within grains) and the etching rate of the substrate as a function of solution composition, $\mathrm{pH}$, and thickness - provide insights into galvanic reaction 
processes with good spatial and temporal resolution that can help towards morphological control of the final nanostructured surface.

\section{References:}

[1] M. Schlesinger, Electroless and Electrodeposition of Silver, Chapter 5 in “Modern Electroplating”, 5th Ed., M. Schlesinger and M. Paunovic, Eds., Wiley (2011).

[2] C. M. Cobley, Y. N. Xia. Material Science and Engineering Report 70 (2010) 44.

[3] C. M. Hangarter et al., Angew. Chem. Int. Ed. Engl. 49 (2010) 7081.

[4] S. W. Chee et al., Chemical Communications 51 (2015) 168.

[5] N. de Jonge and F. M. Ross, Nature Nanotechnology 6 (2011) 695.

[6] F. M. Ross, Science 350 (2015) aaa9886. DOI: 10.1126/science.aaa9886

[7] J. H. Park et al., Microscopy and Microanalysis 19 Suppl. 2 (2012) 1098.

[8] J. H. Park et al., Microscopy and Microanalysis 18 Suppl. 2 (2013) 486.

[9] J. H. Park et al., Microscopy and Microanalysis 21 Suppl. 3 (2015) 1791.

[10] We gratefully acknowledge funding from the National Science Foundation

(NSF-GOALI: DMR-1310639), and the technical assistance of M. C. Reuter and A. W. Ellis.
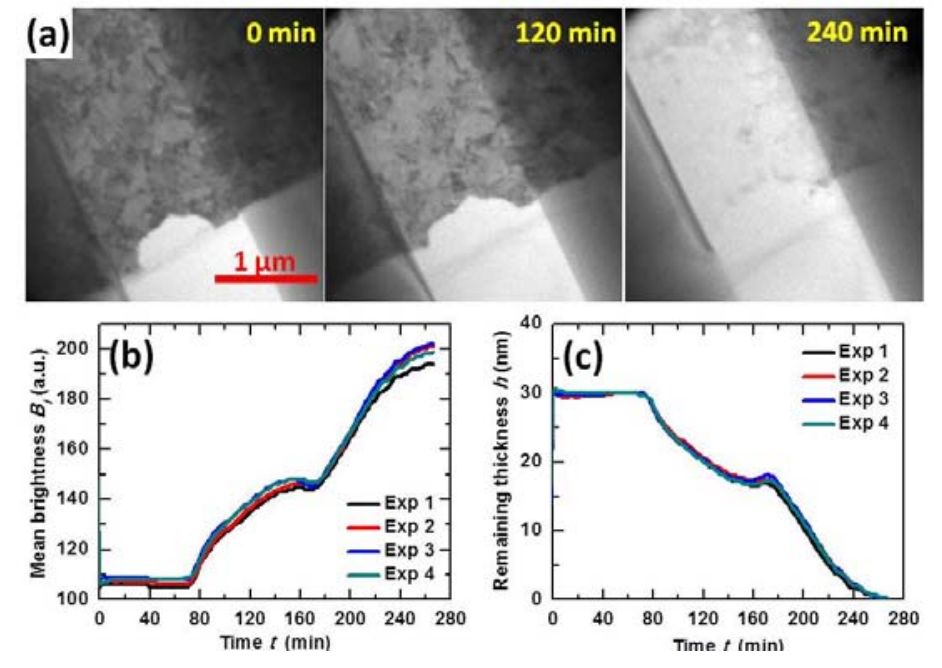

(a)
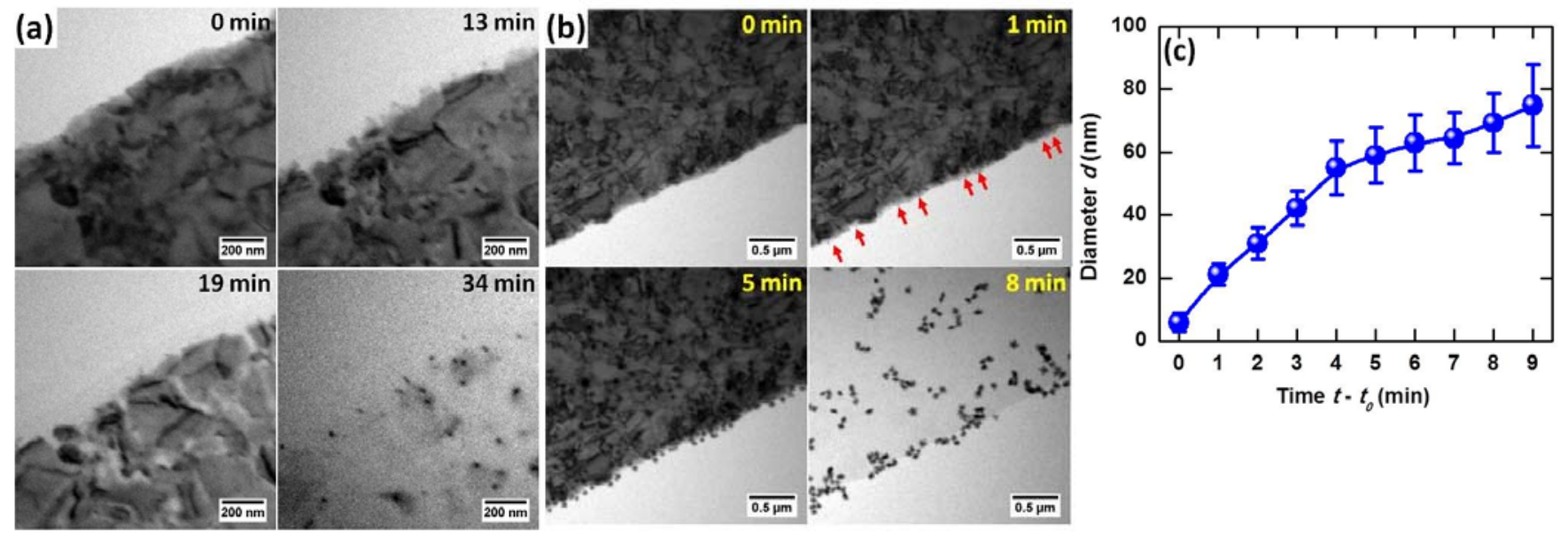

deionized water $(\mathrm{pH}=7$ ) at a flow rate of 5 $\mu \mathrm{l} / \mathrm{min}$ and constant irradiation. (b) Measured brightness changes of $\sim 30 \mathrm{~nm}$ thick Ni film as a function of the irradiation time. Several experiments are shown. (c) Estimated remaining thickness of the $\mathrm{Ni}$ films.

Figure 2. (a) Formation of $\mathrm{Pd}$ particles and simultaneous dissolution of $\mathrm{Ni}$ film in $0.1 \mathrm{M} \mathrm{H}_{2} \mathrm{SO}_{4}(\mathrm{pH}=$ 1) solution with ppm levels of $\mathrm{PdSO}_{4}$ and without electrolyte flow. (b) The same solution at higher flow rate. (c) Size evolution of Pd particles versus time, with to the time at which particles are first visible. 